\title{
Cerebellar Cryptococcoma in an Immunocompetent Adult: A Rare Occurrence Report of a Case and Review of Literature
}

\author{
Manish Kumar ${ }^{1} \quad$ Ankur Bajaj ${ }^{1} \quad$ Manoj Kumar Tewari ${ }^{1}$ \\ ${ }^{1}$ Department of Neurosurgery, Postgraduate Institute of Medical \\ Education \& Research, Chandigarh, India \\ 2Department of Radio-diagnosis and Imaging, Postgraduate Institute \\ of Medical Education \& Research, Chandigarh, India \\ ${ }^{3}$ Department of Histopathology, Postgraduate Institute of Medical \\ Education \& Research, Chandigarh, India
}

\author{
Paramjit Singh ${ }^{2}$ Bishan Das Radotra ${ }^{3}$
}

Indian J Neurosurg:2020;9:204-207

\author{
Address for correspondence Manoj Kumar Tewari, MBBS, MS, MCh, \\ Department of Neurosurgery, Postgraduate Institute of Medical \\ Education \& Research, Chandigarh 160012, India \\ (e-mail: manojktewari@gmail.com).
}

\begin{abstract}
Keywords

- cryptoccocal infection

- cerebellar

cryptococcoma

- neurotropic

- immunocompetent

This study reports a rare case of cryptococcoma of the brain and review the related literature. An intracranial lesion, in a healthy, middle-aged adult who underwent surgery with a preoperative diagnosis of neoplastic/metastatic lesion, which turned out to be cryptococcoma, is being reported. Histopathological examination revealed that this immunocompetent patient had cryptococcoma of the cerebellum. He responded to antifungal chemotherapy (amphotericin B initially for 2 weeks and later oral fluconazole for 10 weeks) very well. Occurrence of cryptococcomas in immunocompetent patients is rare and responds to antifungal chemotherapy very well. Only six cases (including this) have been reported in literature till now.
\end{abstract}

\section{Introduction}

Immunocompromised patients, because of low cell-mediated immunity, are prone to opportunistic infections and one such opportunistic fungal infection is Cryptococcus. It is caused by an encapsulated yeast Cryptococcus neoformans. This infection is usually transmitted via respiratory pathway through the spores found in the powder and bird droppings. Initially, infection is usually a pneumonitis and is self-limited ${ }^{1}$ as in most individuals the immune system is able to eradicate the disease, but sometimes it can lie dormant in granulomatous form. ${ }^{2}$

This infection spreads from the lungs to the central nervous system (CNS) via blood. ${ }^{3}$ It is very uncommon to have this infection in an immunocompetent host. Prior case reports of isolated cryptococcoma have been described in immunocompetent hosts, but in the majority of these cases, the patients were eventually found to have underlying diabetes mellitus or another cause for having previously unrecognized compromised immune status. ${ }^{4,5}$ In this communication, an immunocompetent male patient having a cryptococcoma in the cerebellum, which is a rare location, along with atypical magnetic resonance imaging (MRI) findings has been presented and relevant literature has been reviewed. To our knowledge, this is the sixth case reported in the literature.

\section{Case Report}

A 48-year-old male patient presented with complaints of moderate to severe intensity headache off and on, giddiness and occasional vomiting since 3 months. There was no history of any fever, seizures, or loss of weight. Examination revealed an average built male with bilateral papilloedema and no other focal neurological deficit. His routine hematological and biochemical parameters were within normal limits. There was no history of hypertension, diabetes mellitus, tuberculosis, any other systemic illness, or any history suggesting immunoincompetence. He underwent a plain and contrast MRI of the brain that revealed a $3 \times 3 \times 4$-cm poorly marginated cystic lesion in the left cerebellar hemisphere near the midline with gross surrounding edema causing
Dol https://doi.org/ 10.1055/s-0039-3402591 ISSN 2277-954X. (c) 2020. Neurological Surgeons' Society of India.

This is an open access article published by Thieme under the terms of the Creative Commons Attribution-NonDerivative-NonCommercial-License, permitting copying and reproduction so long as the original work is given appropriate credit. Contents may not be used for commercial purposes, or adapted, remixed, transformed or built upon. (https://creativecommons.org/licenses/by-nc-nd/4.0/)

Thieme Medical and Scientific Publishers Pvt. Ltd., A-12, 2nd Floor, Sector 2, Noida-201301 UP, India 
pressure effect and chinking of the fourth ventricle leading to hydrocephalus. The lesion was hypointense on $\mathrm{T} 1$ and hyperintense on T2-weighted images on MRI. Postcontrast MR images showed minimal heterogeneous enhancement. Diffusion-weighted imaging (DWI) and magnetic resonance spectroscopy (MRS) were also done. MRS revealed increased choline peak at the periphery. No restriction was seen on DWI ( - Fig. 1A-E). Following MR studies, a working diagnosis of metastasis was made. Patient underwent right side ventriculoperitoneal shunt followed by suboccipital craniotomy and gross total excision of mass lesion.

The histopathological examination revealed small pieces of cerebellar tissue containing a relatively well-circumscribed inflammatory lesion. It was composed of small amount of fibro-connective which surrounded numerous yeast forms of cryptococci. The fungal organisms varied in size and were apparent even on hematoxylin-eosin (H\&E)-stained sections ( -Fig. 1F). A large amount of extracellular mucin was apparent around fungi that resulted in a honey-comb appearance. Few budding forms were noted. Sparse lympho-histiocytic response with occasional giant cells were present. The overlying meninges were involved by inflammatory response. Patient was immediately started on intravenous amphotericin B (1 mg/kg/d) and had to switch to fluconazole (400 mg daily) as patient developed amphotericin toxicity.
Drugs were continued for 10 weeks and patient improved clinically and the lesion disappeared as evident from MRI at 5 months of follow-up.

\section{Discussion}

Cryptococcus is an encapsulated yeast of which two varieties, C. neoformans var. neoformans and C. neoformans var. gattii are recognized. The former predominantly infects immuno-compromised patients and causes disseminated opportunistic meningeal infection of the CNS in immunocompromised patients while the latter usually infects immunocompetent patients. ${ }^{6}$ Isolated cerebellar cryptococcoma in an immunocompetent patient is a rare condition. ${ }^{7}$

CNS infection is the second most common site after the lungs for cryptococci as it has a strong neurotropic tendency. Cerebrospinal fluid(CSF)not only provides optimal nutritional environment, but also lacks soluble anticryptococcal factors as compared with serum and this is the cause of serious CNS manifestations. ${ }^{6}$ Common predisposing factors are human immunodeficiency virus (HIV) infection with CD4+ cell count $<200$ cells/ $\mu \mathrm{L}$, long-term steroid therapy, diabetes mellitus, cancer, renal failure, immunologic disease, immunosuppressant treatment, organ transplant, lymphoma, acidosis, and idiopathic CD4 + lymphocytopenia. ${ }^{8}$
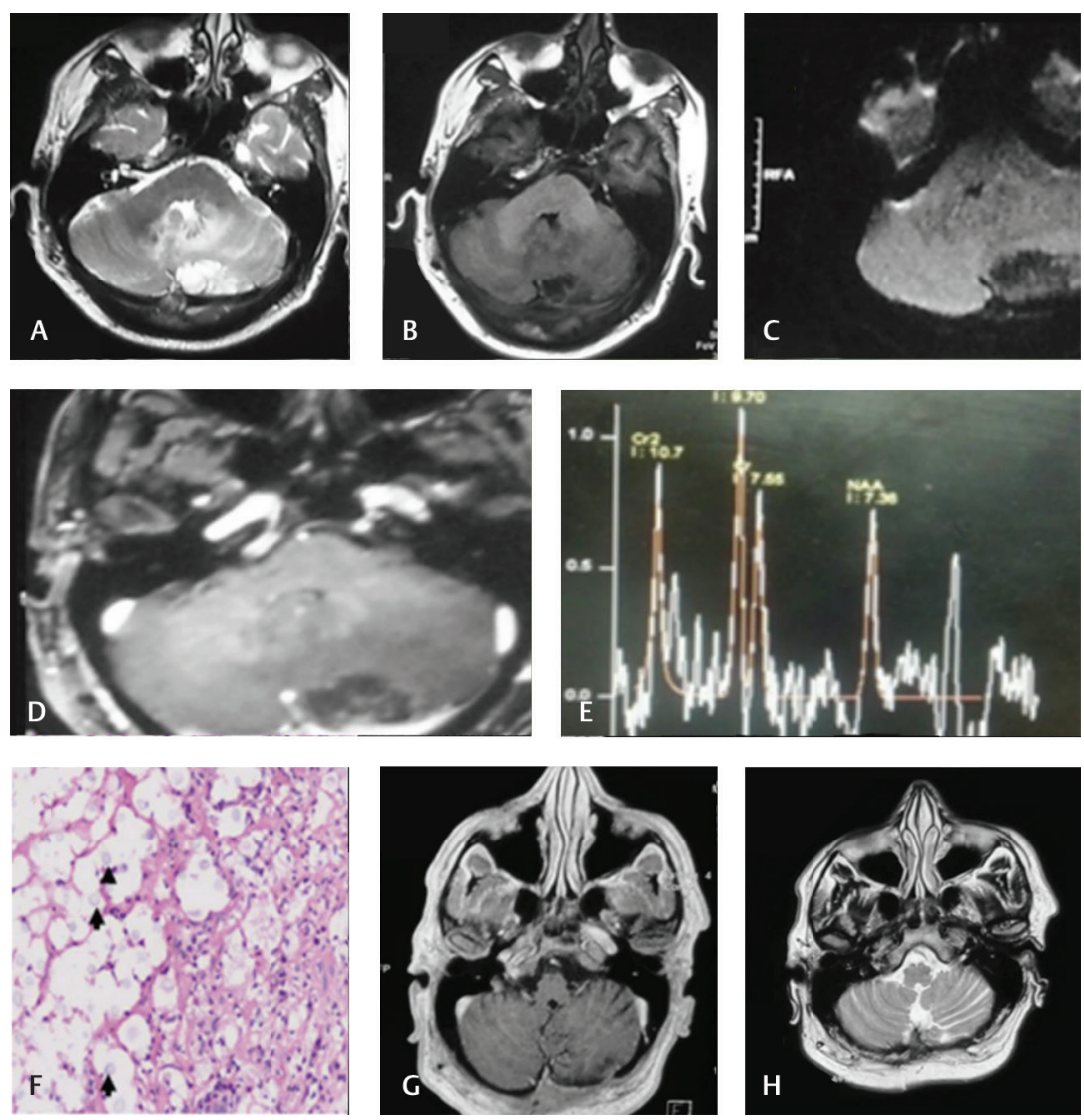

Fig. 1 (A-D) Axial MRI T1 and T2 images showing T2-hyperintense (A) and T1-hypointense lesion in the left cerebellum with edema. There is no diffusion restriction as seen in diffusion (C) maps. Minimal contrast enhancement is noted at the periphery of the lesion (D). Raised choline peak seen on MRS (E). (F) Image shows many cryptococci (arrows) surrounded by mucoid material present in a sparsely inflamed fibroconnective tissue (H\&E. original magnification $\times 20)$. $(\mathbf{G}, \mathbf{H})$ Postoperative-postantibiotic T2 and contrast images show resolution of the lesion. MRI, magnetic resonance imaging; MRS, magnetic resonance spectroscopy. 


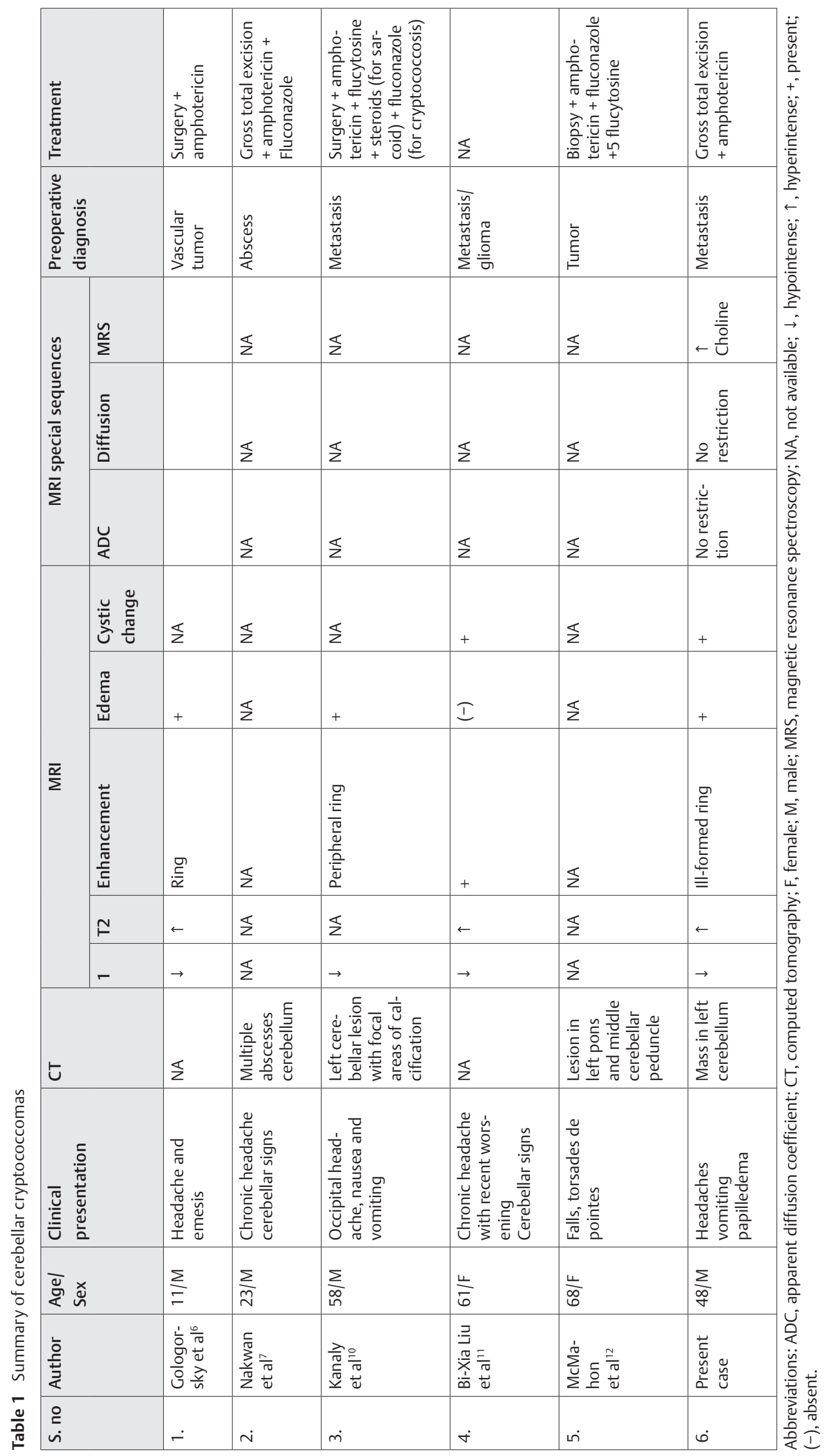


Headache is the most common presentation. Other nonspecific symptoms, such as lethargy, fever, weakness, and anorexia, may also be found. Duration from the onset of symptoms to diagnosis is variable. It is usually longer in the healthier host than in the immunosuppressed host. Headache with increase intracranial pressure for 3 months duration was the presenting complaint of our patient. Cryptococcal CNS infections can present as meningeal or parenchymal forms. Meningitis is the predominant manifestation while parenchymal involvement may be in the form of cryptococcomas, dilated Virchow-Robin spaces, multiple enhancing cortical nodules, or a mixed variety. ${ }^{6,9}$

The diagnosis is challenging in these otherwise healthy patients. The CSF profile shows low glucose, elevated protein, and elevated leukocyte counts, with relative lymphocytosis. Inability to generate adequate inflammatory response CSF in immune-compromised patients may even be normal. Demonstration of Cryptococcus in the CSF using India ink staining clinches the diagnosis. Cryptococcal antigen latex agglutination titers in the CSF and blood are important adjuncts in the diagnosis. CSF cryptococcal antigen is positive in more than $90 \%$ of the cases. ${ }^{6}$ In the present case, histopathological slide shows many cryptococci surrounded by mucoid material ( - Fig. 1F).

Cryptococcomas are usually hypointense on T1 and hyperintense on T2 sequences of MRI with peripheral edema and ring-shaped enhancement after gadolinium injection that too is variable as seen in our case. Advanced sequences on MRI such as DWI, apparent diffusion coefficient, FLAIR (fluid attenuated inversion recovery), and MRS, show no explicit features pathognomonic of neurocryptococcosis. - Table 1 reviews the presentation and MRI brain findings, and treatment details of six cases of cerebellar cryptococcomas known till date. ${ }^{6,710-12}$

Culture of the organism remains the gold standard for diagnosis, with a reported positive rate of almost $100 \%$. Once diagnosed, initial therapy is with intravenous amphotericin $\mathrm{B}$, at 0.7 to $1.0 \mathrm{mg} / \mathrm{kg} /$ day, preferably in combination with oral flucytosine, at $100 \mathrm{mg} / \mathrm{kg} /$ day for 2 weeks or when the India ink stain becomes negative, followed by oral fluconazole at $400 \mathrm{mg} /$ day for a minimum of 10 weeks. CSF should be sampled after 2 weeks of initial therapy to assess for the clearance of fungus. In patients with concomitant HIV infection, lifelong maintenance therapy is achieved after induction therapy by administering fluconazole at 200 to $400 \mathrm{mg} /$ day. $^{6}$
To conclude, cerebellar cryptococcoma may be considered as differential diagnosis even in immunocompetent patient and once diagnosed it is a curative disease.

\section{Funding}

None.

\section{Conflict of Interest}

None declared.

\section{References}

1 Singer EJ, Valdes-Sueiras M, Commins D, Levine A. Neurologic presentations of AIDS. Neurol Clin 2010;28(1):253-275

2 Jarvis JN, Harrison TS. HIV-associated cryptococcal meningitis. AIDS 2007;21(16):2119-2129

3 Kumari R, Raval M, Dhun A. Cryptococcal choroid plexitis: rare imaging of the CNS findings of Cryptococcus in immunocompetent individual. Br J Radiol 2010;83(985):e14-e17

4 Kesler R, Maertens P. Pontine cryptococcoma in a nonimmunocompromised individual: MRI characteristics. J Neuroimaging 1999;9(2):118-121

5 Saigal G, Post MJ, Lolayekar S, Murtaza A. Unusual presentation of central nervous system cryptococcal infection in an immunocompetent patient. AJNR Am J Neuroradiol 2005;26(10):2522-2526

6 Gologorsky Y, DeLaMora P, Souweidane MM, Greenfield JP. Cerebellar cryptococcoma in an immunocompetent child. Case report. J Neurosurg 2007;107(4, Suppl):314-317

7 Nakawan N, Songjamrat A, Tungsinmonkong K, Nakwan N. Cerebellar cryptococcoma in an immunocompetent adult. Southeast Asian J Trop Med Public Health 2009;40:1034-1037

8 Khwaja GA, Ranjan R, Gupta M, Chowdhury D, Kushwaha R. Long term outcome of intracranial and cutaneous cryptococcal infection in an immunocompetent man. JIACM 2010;11(4):319-325

9 Ecevit IZ, Clancy CJ, Schmalfuss IM, Nguyen MH. The poor prognosis of central nervous system cryptococcosis among nonimmunosuppressed patients: a call for better disease recognition and evaluation of adjuncts to antifungal therapy. Clin Infect Dis 2006;42(10):1443-1447

10 Kanaly CW, Selznick LA, Cummings TJ, Adamson DC. Cerebellar cryptococcoma in a patient with undiagnosed sarcoidosis: case report. Neurosurgery 2007;60(3):E571, discussion E571

11 Liu BX, Dai XJ, Liu H, Gong HH, Wang YX, Zhang LL. Cerebellar cryptococcosis characterized by a space-occupying lesion in an immunocompetent non-HIV patient. Neuropsychiatr Dis Treat 2014;11:21-24

12 McMahon JH, Grayson ML. Torsades de pointes in a patient receiving fluconazole for cerebral cryptococcosis. Am J Health Syst Pharm 2008;65(7):619-623 